INGENIERÍA CIVIL

\title{
Incertidumbre paramétrica en modelos dinámicos de estructuras civiles
}

\author{
Claudia P. Moreno"s, Peter Thomson* \\ *Escuela de Ingeniería Civil y Geomática, Universidad del Valle, Cali. Grupo de Investigación en \\ Ingeniería Sísmica, Ingeniería Eólica y Estructuras Inteligentes, G-7. \\ §email: clamoren@univalle.edu.co
}

(Recibido Marzo 16 de 2010- Aceptado: Mayo 31 de 2010)

\begin{abstract}
Resumen
El análisis dinámico es un procedimiento esencial para diseñar una estructura sujeta a cargas dinámicas. Su objetivo es determinar la respuesta estructural e interpretar los resultados teóricos para obtener un diseño adecuado para la estructura. Sin embargo, la existencia de incertidumbres asociadas a parámetros geométricos, propiedades de materiales y dinámica no modelada, que afectan la respuesta dinámica de la estructura, actualmente no son consideradas en los modelos estructurales. Aunque se han desarrollado diferentes herramientas para el tratamiento de incertidumbre en sistemas, la incertidumbre estructurada resulta idónea para ser incorporada en modelos estructurales debido a que permite modelar las variaciones tanto en parámetros físicos como en la dinámica no considerada en los sistemas dinámicos. Este trabajo presenta una descripción de incertidumbre paramétrica para un modelo de tribuna metálica construido en la Escuela de Ingeniería Civil y Geomática de la Universidad del Valle. Los resultados muestran que el modelo analítico con incertidumbre es capaz de representar un conjunto de modelos identificados a partir de ensayos de vibración forzada mediante la variación de los parámetros de masa y amortiguamiento.
\end{abstract}

Palabras Claves: Modelación de incertidumbre, Dinámica estructural, Descripciones de incertidumbre, Identificación de sistemas.

CIVIL ENGINEERING

\section{Parametric uncertainty in dynamic models of civil structures}

\begin{abstract}
Dynamic analysis of a structure is an essential procedure to design structures subjected to dynamic loads. The objective of dynamic analysis is to determine the structure's response and interpret those results in order to design the structure. However, the presence of uncertainties associated with geometric parameters, material properties and unmodelled dynamics affects the structural response, and these uncertainties are not considered in the structure's dynamical model. Although several tools for treatment of uncertainty have been developed, structured uncertainty is ideal for inclusion into structural models because it allows modelling the variations in both physical parameters and neglected dynamics in systems. This paper presents a parametric uncertainty description for a steel grandstand model built at School of Civil Engineering and Geomatics, Universidad del Valle. Results show that analytical uncertainty model of the structure is able to represent a set of dynamical systems identified by forced vibration tests under different occupation conditions with variations in mass and damping parameters.
\end{abstract}

Keywords: Modeling uncertainty, Structural dynamics, Uncertainty descriptions, System identification. 


\section{Introducción}

Los modelos dinámicos de estructuras civiles son derivados de las ecuaciones de movimiento de la mecánica newtoniana. Estos modelos son creados con el fin de predecir y describir el comportamiento dinámico de las estructuras. Sin embargo, un modelo que describa exactamente el comportamiento de un sistema real no puede ser construido. Por consiguiente, el modelo es en cierto nivel una aproximación del comportamiento del sistema físico, y su calidad depende de la exactitud entre la respuesta calculada y la respuesta medida del sistema. Estas diferencias entre respuestas son referidas como incertidumbres, las cuales pueden ser modeladas de diferentes maneras y su descripción matemática depende de la herramienta utilizada para el análisis de los sistemas. Algunas herramientas utilizadas para la representación y análisis de incertidumbre en modelos han sido los métodos estocásticos, sensibilidad diferencial, multi-modelos, álgebra de intervalos y conjuntos difusos.

En el enfoque estocástico, la incertidumbre es modelada por variables aleatorias o procesos aleatorios, lo cual requiere de información estadística para estimar las funciones de distribución probabilísticas que describen la variabilidad de los parámetros y consideraciones de riesgo. Ahora, cuando la información estadística no está disponible y los límites de la incertidumbre no son conocidos, resulta práctico analizar el sistema ante pequeñas variaciones en los parámetros. Este procedimiento es llamado sensibilidad diferencial, y es definido por derivadas parciales de primer orden con respecto a un parámetro (Weinmann, 1991).

En el marco del álgebra de intervalos, la incertidumbre en los elementos es representada por un conjunto cerrado de parámetros que pueden variar entre los valores extremos de intervalos, por tanto, los sistemas deben ser analizados utilizando operaciones entre intervalos (Modarreszadeh, 2005). En el marco de la lógica difusa la incertidumbre es expresada por conjuntos difusos, cuyos elementos tienen grados de pertenencia descritos por funciones de pertenencia valoradas en el intervalo real entre 0 y 1 . El dominio de la función es conocida como el intervalo de confianza y el rango como el nivel de suposición (Modarreszadeh, 2005). Por otra parte, el enfoque de multi-modelos está basado en la existencia de diferentes modelos para describir un sistema. En este, no se requiere asignar una probabilidad o credibilidad a los valores de los parámetros del modelo para evaluar la incertidumbre, ya que los modelos se obtienen directamente de datos experimentales (Monte, 2009).

En estructuras civiles, la incertidumbre ha sido reconocida en las metodologías de diseño basadas en la confiabilidad y cálculo del riesgo de falla, y en el ajuste de modelos de elementos finitos basados en datos de vibración. El objetivo general del ajuste de modelos es la generación de modelos confiables a través de ajustes paramétricos en los mismos, de tal forma que se minimice la diferencia entre las predicciones analíticas y los resultados experimentales (Vélez et al., 2009). Este método ha sido usado en la identificación de daños estructurales y cambios de frecuencias naturales, principios básicos del monitoreo de salud estructural.

Adicionalmente, técnicas probabilísticas han sido fusionadas con el método de los elementos finitos, dando lugar al método de elementos finitos estocástico (Haldar \& Sankaran, 2000). En éste, las variables que representan las cargas, el módulo de elasticidad y las propiedades geométricas son caracterizadas por distribuciones de probabilidad o por distribuciones aleatorias espaciales. Además se introduce el concepto de función de desempeño donde se establecen criterios y límites que el sistema debe satisfacer. El método estocástico ha estado presente en la caracterización de matrices de rigidez para vigas Euler-Bernoulli aleatorias, estimación de la variabilidad de la respuesta a excitaciones estacionarias y transitorias determinísticas y el desarrollo de métodos de simulación para examinar cuantitativamente la influencia sobre la respuesta estructural de varios modelos de densidad probabilística para campos de sistemas aleatorios (Gupta, 2000). 
De igual forma, el análisis de intervalos ha sido incorporado al método de elementos finitos donde el objetivo es encontrar los límites superior e inferior de los diferentes parámetros del modelo y utilizar estos resultados en el proceso de diseño. Durante la última década, investigaciones sobre el problema de intervalos de valores propios han sido realizadas enérgicamente, proponiendo diferentes métodos que permiten encontrar los límites de las frecuencias naturales del sistema (Modarreszadeh, 2005).

Por otra parte, en el marco del control óptimo y robusto se ha desarrollado una herramienta poderosa, denominada transformación lineal fraccional, para estudiar sistemas con incertidumbre en el dominio de la frecuencia. Esta herramienta, introducida por Doyle (Zhuo et al., 1991) aproximadamente 20 años atrás, utiliza perturbaciones complejas estructuradas alrededor de un modelo nominal. Dichas perturbaciones consideran no solo variaciones en parámetros físicos sino también la dinámica no modelada de los sistemas, y son organizadas en un arreglo matemático diagonal de norma limitada lo cual hace más eficiente el cálculo computacional. Adicionalmente, esta formulación reconoce la presencia de incertidumbre durante todo el proceso de análisis de los sistemas y garantiza el desempeño deseado para todo el conjunto de modelos descritos, siendo esta su principal ventaja ante las demás herramientas utilizadas para el tratamiento de incertidumbre.

Debido a estas ventajas, el presente trabajo propone la expresión de los modelos dinámicos de estructuras civiles como transformaciones lineales fraccionales que permitan el tratamiento y análisis de los sistemas con parámetros de incertidumbre. Para esto, se presenta una descripción de las posibles fuentes de incertidumbre y los fundamentos básicos de su representación matemática, y finalmente se obtiene un modelo con incertidumbre para una estructura metálica tipo tribuna construida en la Escuela de Ingeniería Civil y Geomática de la Universidad del Valle a partir de un modelo analítico y datos experimentales de vibración.

\section{Fuentes de incertidumbre en estructuras civiles}

Universalmente el método de los elementos finitos es utilizado como herramienta para analizar el comportamiento dinámico de sistemas estructurales. Sin embargo es bien sabido que existen discrepancias entre las respuestas calculadas por dichos modelos y las respuestas reales de los sistemas físicos. Para estructuras civiles en particular, estas variaciones se encuentran relacionadas con los coeficientes de masa, amortiguamiento y rigidez del modelo estructural y son originadas por diferentes fuentes, algunas de ellas se mencionan a continuación.

Variaciones en la masa de la estructura son generadas por las condiciones de ocupación y uso de la estructura que no son constantes a lo largo de su vida útil (Reynolds et al., 2004) (Moncayo et al., 2006). Por otra parte, el amortiguamiento de una estructura resulta muy difícil de determinar debido a los numerosos mecanismos que contribuyen a la disipación de energía, entre los que se pueden incluir la fricción entre conexiones, fricción entre elementos no estructurales y grietas (Chopra, 1995). Por tal motivo, con el fin de simplificar el modelo, la propiedad de amortiguamiento en la estructura es representada por un amortiguador viscoso lineal. En general, la fuente de incertidumbre más grande se encuentra en la cuantificación de este parámetro.

Por último, la variación en la rigidez puede ser atribuida a las propiedades de los materiales asumidos en la creación del modelo estructural, que pueden cambiar en cada miembro de la estructura. La interacción suelo-estructura, usualmente no modelada, también afecta notablemente la rigidez de las estructuras. De acuerdo a Trifunac et al. (2001), las no linealidades del suelo pueden generar una reducción del 64\% de las frecuencias naturales cuando las amplitudes del movimiento sísmico son considerables.

Igualmente, existen otros factores generales que pueden generar incertidumbre en la modelación de estructuras civiles. Por ejemplo, los elementos no 
estructurales, representados por paredes y divisiones, usualmente se suponen independientes de la estructura para la modelación pero en la construcción terminada aportan al sistema de resistencia.

Otro tipo de incertidumbre en estructuras se refiere a las diferencias entre la respuesta calculada por el modelo parametrizado y la respuesta real del sistema. En esta se incluyen la dinámica no modelada y las no-linealidades presentes en el sistema así como también el ruido producido por los sensores de medición.

La dinámica no modelada del sistema generalmente se incluye debido a componentes no estructurales, variaciones en las conexiones de vigas y columnas, y la interacción sueloestructura. Estos componentes con frecuencia tienen un comportamiento no lineal cuando están sujetos a excitaciones sísmicas, aun si los miembros estructurales no han fluido (May, 1997). Ahora, la incertidumbre generada por los sensores utilizados en la medición de la respuesta estructural es mínima comparada con las otras fuentes de incertidumbre. Esto se debe a que la precisión de los instrumentos para medir las vibraciones en estructuras civiles es menor del 1\% (May, 1997).

En aplicaciones sísmicas, la magnitud, duración y contenido de frecuencias del movimiento en la base es difícil de cuantificar, lo cual genera gran incertidumbre. Esta incertidumbre no está directamente relacionada con el modelo pero de igual forma debe incorporarse en el análisis de sistemas, especialmente en estructuras civiles donde la excitación externa es usualmente más incierta que el propio modelo. Estos parámetros pueden ser medidos en cierta forma al modelar las condiciones de sitio locales, tales como la cercanía a fallas, propiedades del suelo y en general la sismicidad de la región (May, 1997).

\section{Modelo dinámico estructural}

Los modelos de estructuras civiles generalmente son representados por ecuaciones diferenciales ordinarias de orden finito. Para el caso de un sistema sujeto a excitaciones dinámicas, las ecuaciones diferenciales que lo describen son (Moreno et al. 2010).

$M \frac{d^{2} u}{d t^{2}}+C \frac{d u}{d t}+K u=P(t)$

Donde $M, C y K$ representan las matrices de masa, amortiguamiento y rigidez, respectivamente. $u$ representa el vector de desplazamiento, $d u / d t$ el vector de velocidad, $d^{2} u / d t^{2}$ el vector de aceleraciones y $P(t)$ el vector la excitación externa.

La relación entre las entradas y salidas de un sistema lineal invariante en el tiempo puede ser representada por el diagrama de bloques mostrado en la Figura 1.

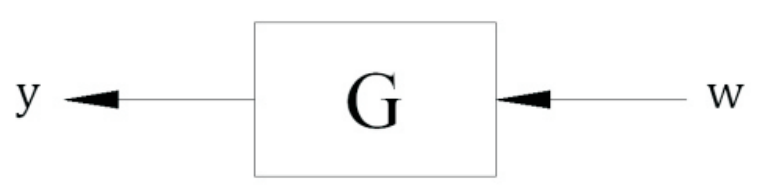

Figura 1. Representación de sistemas lineales

Donde $w$ representa las entradas del sistema, $y$ representa las salidas del sistema y $G$ representa el modelo del sistema. Cuando el modelo es descrito por un conjunto de ecuaciones lineales de primer orden es conocido como modelo en el espacio de estados, y es expresado de la siguiente forma:

$$
G:=\left[\frac{A \mid B}{C \mid D}\right]=\left\{\begin{array}{l}
\dot{x}=A x+B w \\
y=C x+D w
\end{array}\right\}
$$

En esta expresión, $x$ es llamado vector de estados, $A$ es la matriz de estados, $B$ es la matriz de entrada, $C$ la matriz de salida y $D$ la matriz de transmisión directa. 
Al definir las variables de estado $x$ y el vector de entradas $y$, como:

$$
\begin{gathered}
x=\left[\begin{array}{ll}
u & \frac{d u}{d t}
\end{array}\right]^{\prime} \\
w=P(t)
\end{gathered}
$$

Se obtienen las siguientes matrices que conformarán el espacio de estados,

$$
\begin{gathered}
A=\left[\begin{array}{cc}
0 & I \\
-M^{-1} K & -M^{-1} C
\end{array}\right] \\
B=\left[\begin{array}{c}
0 \\
M^{-1}
\end{array}\right]
\end{gathered}
$$

Las salidas del sistema se pueden tomar como desplazamientos, velocidades o aceleraciones totales o relativas de la estructura, dependiendo de la respuesta que se quiera visualizar. En caso de medir las aceleraciones del sistema, la matriz de salida y de transmisión directa toman la forma,

$$
\begin{gathered}
C=\left[\begin{array}{cc}
M^{-1} K & -M^{-1} C
\end{array}\right] \\
D=\left[M^{-1}\right]
\end{gathered}
$$

De esta manera, se obtiene el modelo dinámico estructural en el espacio de estados, cuya representación resulta muy útil para el tratamiento de las incertidumbres presentes en el sistema.

\section{Representación de sistemas con incertidumbre}

La idea básica para modelar sistemas con incertidumbre es separar las variables que se conocen de las que son desconocidas en un ciclo realimentado y poner límites de posibles valores a los elementos desconocidos. Para esto, la transformación lineal fraccional, en inglés linear fractional transformation (LFT), resulta ser una herramienta poderosa y flexible (Balas et al., 2001).

Zhuo (1991) introdujo el concepto de LFT con el fin de examinar la robustez de los sistemas de control dinámicos bajo incertidumbres estructuradas. Una LFT es una interconexión de operadores ordenados en una configuración retroalimentada representada generalmente por un diagrama de bloques entrada-salida.

La Figura 2 muestra una LFT con realimentación superior.

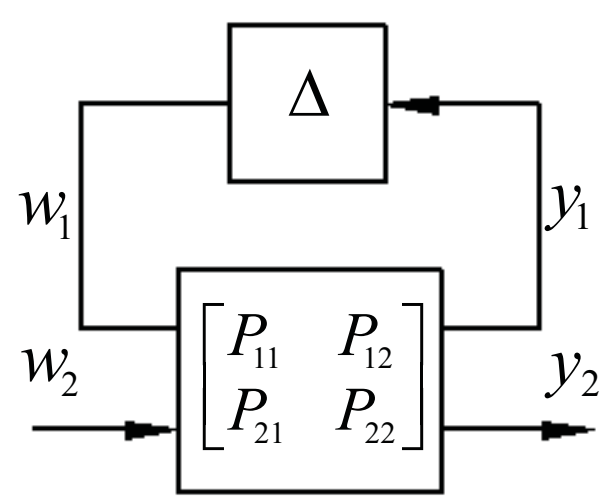

Figura 2. Transformación lineal fraccional superior

Matemáticamente esta LFT se expresa por un conjunto de ecuaciones tal que,

$$
\left[\begin{array}{l}
y_{1} \\
y_{2}
\end{array}\right]=\left[\begin{array}{ll}
P_{11} & P_{12} \\
P_{21} & P_{22}
\end{array}\right]\left[\begin{array}{l}
w_{1} \\
w_{2}
\end{array}\right]
$$

$$
w_{1}=\Delta y_{1}
$$

La solución para este conjunto de ecuaciones está dada por

$$
\begin{gathered}
y=\left(P_{22}+P_{21} \Delta\left(I-P_{11}\right)^{-1} P_{12}\right) w \\
y=F_{u}(P, \Delta) w
\end{gathered}
$$


Análogamente, una LFT con realimentación inferior tiene la forma

$$
\begin{gathered}
y=\left(P_{11}+P_{12} \Delta\left(I-P_{22}\right)^{-1} P_{21}\right) w \\
y=F_{L}(P, \Delta) w
\end{gathered}
$$

Físicamente la LFT se puede interpretar como la función de transferencia del sistema con incertidumbre donde el operador $P$ representa el modelo generalizado y $\Delta$ representa las incertidumbres del sistema, las cuales se formulan como un conjunto de operadores de norma limitada $|\Delta| \leq 1$. De esta forma se asume que la planta real existe dentro de la familia de modelos descritos por la LFT. Por otra parte, el bloque de incertidumbre $\Delta$ puede estar compuesto por las dos clases de incertidumbre que se describen en la siguiente sección.

\section{Descripciones de incertidumbre}

Las descripciones de incertidumbre determinan la relación entre el comportamiento deseado del sistema y la robustez ante cambios o perturbaciones en el mismo. Una descripción de incertidumbre demasiado conservativa limita el desempeño del sistema al incluir posibles sistemas que no alcancen el comportamiento deseado. Por esta razón se requieren límites de incertidumbre ajustados que mejoren el desempeño de los sistemas (Balas, 1990).

En el marco de la incertidumbre estructurada, el conjunto de posibles modelos puede ser descrito de dos formas, mediante variaciones paramétricas y/o funciones de peso en del dominio de la frecuencia. Para explicar estas descripciones se utiliza la siguiente notación (Skogestad \& Postlethwaite, 2003):

$\Pi$ : Conjunto de posibles modelos perturbados. $G(s) \in \Pi$ : Modelo nominal sin incertidumbre. $G_{p}(s) \in \Pi$ : Modelos particulares perturbados.

$\Delta$ : Incertidumbre de norma limitada.

\subsection{Descripciones de incertidumbre en el dominio de frecuencia}

Las descripciones de incertidumbre en el dominio de frecuencia permiten tener en cuenta la variación en los datos experimentales en ciertas frecuencias causadas por la dinámica no modelada y no linealidades presentes en el sistema. Esta incertidumbre es representada por una función de peso limitada en frecuencia, $W$, y un bloque de incertidumbre de norma limitada $\Delta$. Sin embargo, puede ser descrita en diferentes formas como se observa a continuación.

\subsubsection{Incertidumbre aditiva}

La incertidumbre aditiva puede usarse para tener en cuenta la dinámica no modelada. En estructuras civiles es causada por la aparición de modos en baja o alta frecuencia que no son reproducidos por el modelo (Balas, 1990). La incertidumbre aditiva es expresada en la Ec. (13)

$$
\begin{gathered}
\Pi_{a}: G_{p}(s)=G(s)+W_{A}(s) \Delta(s) \\
|\Delta(j \omega)| \leq 1 \quad \forall \omega
\end{gathered}
$$

De esta forma, la incertidumbre aditiva envuelve el modelo nominal, tal como se muestra en la Figura 3.

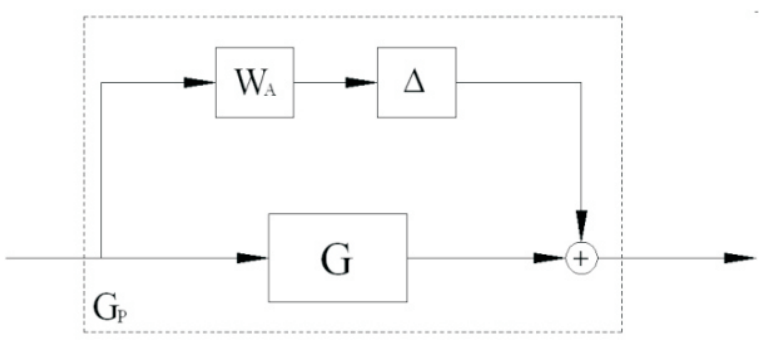

Figura 3. Diagrama de incertidumbre aditiva

\subsubsection{Incertidumbre multiplicativa}

La incertidumbre multiplicativa es usada para representar variaciones relativas en las señales de entrada o salida y es útil para describir los errores de actuadores a alta frecuencia y su dinámica no modelada (Balas, 1990). La incertidumbre 
multiplicativa es utilizada para modelar actuadores y sensores, independientes o combinados, para tener en cuenta las variaciones en las entradas o salidas, representadas en una función de transferencia. En estructuras civiles, esta situación es representada por los dispositivos de control activo utilizados para reducir vibraciones excesivas y en pruebas de sensores que miden la respuesta estructural con un porcentaje de error que puede variar a través de la frecuencia.

En este caso el conjunto de posibles plantas se expresa matemáticamente en la Ec. (14) y gráficamente como se muestra en la Figura 4.

$\Pi_{m}: G_{p}(s)=G(s)\left(I+W_{M}(s) \Delta(s)\right)$

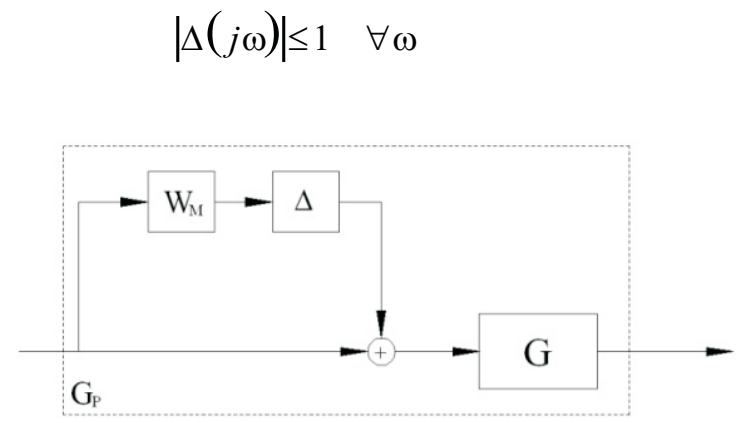

Figura 4. Diagrama de incertidumbre multiplicativa

\subsubsection{Incertidumbre inversa}

Además de la incertidumbre aditiva y multiplicativa, existen otras descripciones de incertidumbre en el dominio de la frecuencia. Estas incertidumbres tienen en cuenta variaciones en la dinámica del sistema e incertidumbre de baja frecuencia y pueden expresarse en las siguientes formas:

$$
\begin{gathered}
\Pi_{i}: G_{p}(s)=\left(I+W_{M}(s) \Delta(s)\right)^{-1} G(s) \\
|\Delta(j \omega)| \leq 1 \quad \forall \omega
\end{gathered}
$$

$$
\begin{gathered}
\Pi_{i}: G_{p}(s)=G(s)\left(I+W_{M}(s) \Delta(s)\right)^{-1} \\
|\Delta(j \omega)| \leq 1 \quad \forall \omega \\
\Pi_{a}: G_{p}(s)=\left(G(s)^{-1}+W_{A}(s) \Delta(s)\right)^{-1} \\
|\Delta(j \omega)| \leq 1 \quad \forall \omega
\end{gathered}
$$

Las Figuras 5, 6 y 7 muestran los diagramas de bloque para las distintas incertidumbres inversas descritas anteriormente.

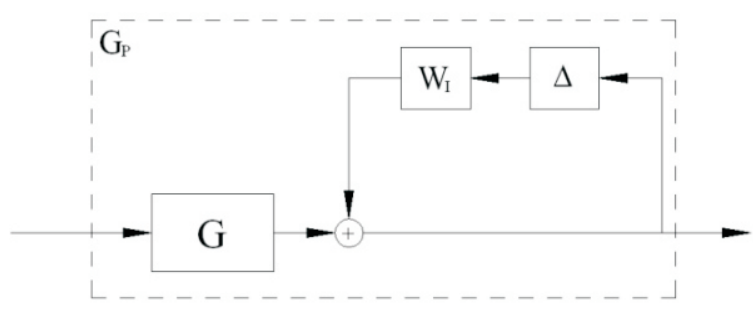

Figura 5. Incertidumbre multiplicativa inversa en la salida

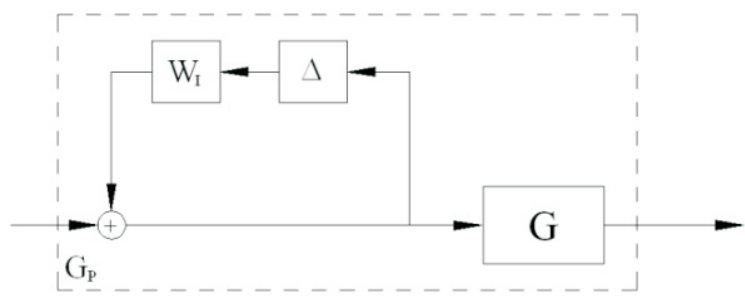

Figura 6. Incertidumbre multiplicativa inversa en la entrada

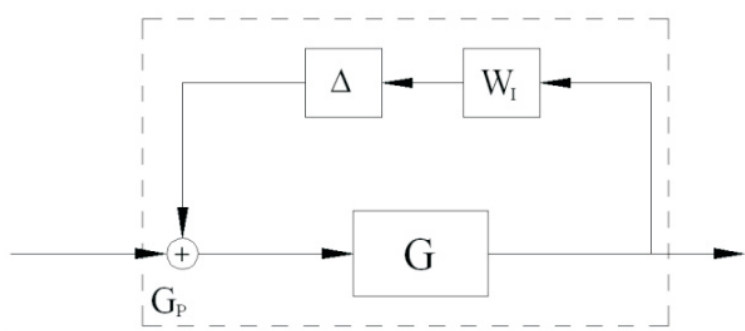

Figura 7. Incertidumbre inversa aditiva 


\subsection{Descripciones de incertidumbre paramétrica}

La incertidumbre paramétrica se cuantifica asumiendo que cada parámetro está limitado en alguna región $\left[\alpha_{\min }, \alpha_{\max }\right]$, teniendo un conjunto de parámetros de la forma (Skogestad \& Postlethwaite, 2003):

$$
\alpha_{p}=\bar{\alpha}\left(1+r_{\alpha} \Delta\right)
$$

donde $\bar{\alpha} \quad$ es el valor promedio y $r_{\alpha}$ la incertidumbre relativa en el parámetro, calculada por

$r_{\alpha}=\frac{a_{\max }-a_{\min }}{a_{\max }+a_{\min }}$

Una propiedad de esta descripción, es que las incertidumbres en los coeficientes de modelos están relacionadas con variaciones reales, es decir, que el bloque de incertidumbre está limitado por el rango de valores reales, $|\Delta| \leq 1$. Debido a que la incertidumbre paramétrica real no puede ser manejada directamente por los métodos de análisis en frecuencia, es necesario formular estas variaciones como incertidumbres complejas. Una aproximación para esto, es limitar las variaciones reales por una variación compleja, sin embargo esta aproximación resulta conservadora al introducir posibles sistemas que no están presentes en el conjunto original de modelos. Por lo tanto, resulta de gran importancia seleccionar una adecuada variación compleja que se ajuste a las variaciones reales (Balas, 1990).

Una alternativa para obtener la descripción compleja es encontrar para cada frecuencia el radio más pequeño que incluye todas las posibles plantas del conjunto (Skogestad \& Postlethwaite, 2003). Para una descripción de incertidumbre aditiva, el radio es

$l_{A}(\omega)=\max _{G_{p} \in \Pi}\left|G_{p}(j \omega)-G(j \omega)\right|$

$\left|W_{A}(j \omega)\right| \geq l_{A}(\omega)$
Para el caso de una descripción de incertidumbre multiplicativa, el radio es

$$
\begin{gathered}
l_{M}(\omega)=\max _{G_{p} \in \Pi}\left|\frac{G_{p}(j \omega)-G(j \omega)}{G(j \omega)}\right| \\
\left|W_{M}(j \omega)\right| \geq l_{M}(\omega)
\end{gathered}
$$

\subsubsection{Incertidumbre en el espacio de estados}

La incertidumbre paramétrica puede ser expresada también en el modelo de espacio de estados, tal que:

$$
G=G_{0}+\sum_{i}^{m} \delta_{i} G_{i}=\left[\begin{array}{cc}
A_{0} & B_{0} \\
C_{0} & D_{0}
\end{array}\right]+\sum_{i}^{m} \delta_{i}\left[\begin{array}{cc}
A_{i} & B_{i} \\
C_{i} & D_{i}
\end{array}\right]
$$

Siendo $m$ el número de parámetros de incertidumbre y $r$ el rango de $G$, se puede factorizar cada una de las matrices de incertidumbre relacionadas como sigue:

$\left[\begin{array}{cc}A_{i} & B_{i} \\ C_{i} & D_{i}\end{array}\right]=\left[\begin{array}{l}E_{i} \\ F_{i}\end{array}\right]\left[\begin{array}{ll}H_{i} & I_{i}\end{array}\right]$

Donde $E_{i}, F_{i}$, tienen $r$ columnas y $H_{i}, I_{i}$, tienen $r$ filas, y el sistema puede ser representado como una LFT de $G_{i}$ respecto a $\Delta$, expresada como:

$$
\begin{aligned}
& {\left[\begin{array}{c}
\dot{x} \\
y \\
z_{1} \\
\vdots \\
z_{m}
\end{array}\right]=\left[\begin{array}{ccccc}
A_{0} & B_{0} & E_{1} & \cdots & E_{m} \\
C_{0} & D_{0} & F_{1} & \cdots & F_{m} \\
H_{1} & I_{1} & 0 & \cdots & 0 \\
\vdots & \vdots & \vdots & \ddots & \vdots \\
H_{m} & I_{m} & 0 & \cdots & 0
\end{array}\right]\left[\begin{array}{c}
x \\
w \\
v_{1} \\
\vdots \\
v_{m}
\end{array}\right]} \\
& \Delta=\operatorname{diag}\left[\delta_{1} I_{r 1}, \ldots \delta_{m} I_{r m}\right]
\end{aligned}
$$




\section{Caso de aplicación: estructura metálica tipo tribuna}

Se realiza un análisis de incertidumbre a una estructura metálica tipo tribuna conformada por dos pórticos separados $2 \mathrm{~m}$. Cada pórtico posee dos columnas que forman una $\mathrm{V}$, esto con el objetivo de replicar la geometría del Estadio Olímpico Pascual Guerrero (Moncayo et al 2006). La viga que une las columnas tiene una longitud de $3.6 \mathrm{~m}$, de los cuales $1.5 \mathrm{~m}$ quedan en voladizo en ambos extremos. Adicionalmente, los pórticos son unidos lateralmente por 5 viguetas en forma de $\mathrm{T}$ que a su vez cumplen la función de los peldaños de la tribuna. Un esquema de la estructura descrita es mostrado en la Figura 8. Este tipo de estructura es seleccionada debido a que estudios previos han comprobado que escenarios públicos como estadios, coliseos y auditorios tienen una gran variación en las propiedades dinámicas producida por el movimiento de las personas (Ortíz et al. 2007). Con el objetivo de tener en cuenta las variaciones de la estructura representadas por su ocupación, se presenta a continuación un modelo con incertidumbre paramétrica que incorpora dichas variaciones.

\subsection{Identificación de sistemas dinámicos}

Con el objetivo de observar la variación de la respuesta de la estructura construida como plataforma experimental, se realizan ensayos de vibración forzada para diferentes condiciones de ocupación mediante un excitador de masa ubicado en la parte central del primer peldaño. La recolección de datos experimentales se realiza mediante el sistema de adquisición portátil de la Escuela de Ingeniería Civil y Geomática, que consta de 8 acelerómetros, acondicionadores de señal y una tarjeta de adquisición de datos. La Figura 9 muestra la plataforma experimental.

Los registros de aceleración se adquieren a una tasa de muestreo de $256 \mathrm{~Hz}$. Las señales de entrada seleccionadas para los ensayos de vibración forzada corresponden a barridos lineales entre 0$10 \mathrm{~Hz}$ ejecutado entre 10-15 min. Se realizan en total 20 ensayos de vibración forzada repartidos en ensayos para las diferentes condiciones de carga que son la tribuna vacía y la tribuna con una, dos y tres personas sentadas y paradas, ubicadas en diferentes posiciones.

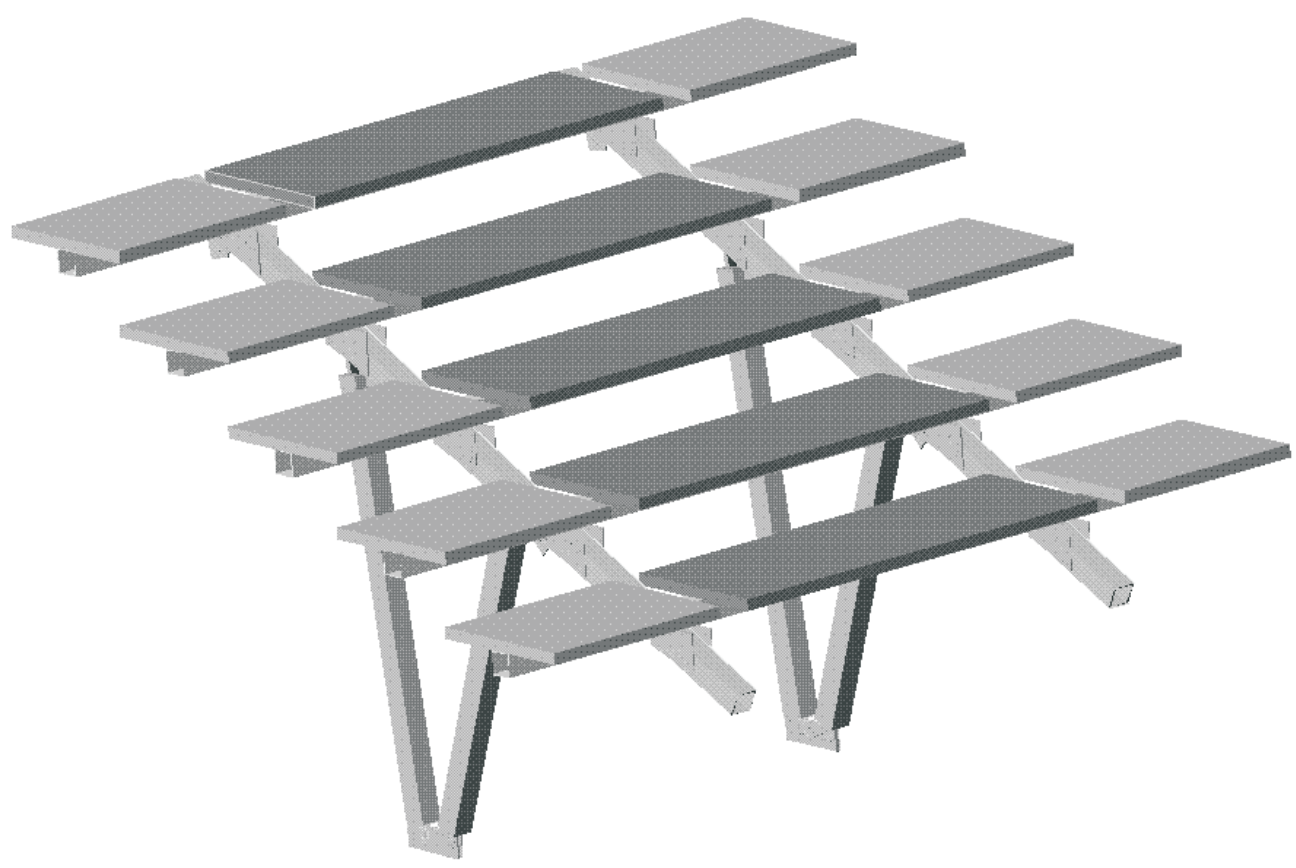

Figura 8. Estructura metálica tipo tribuna 


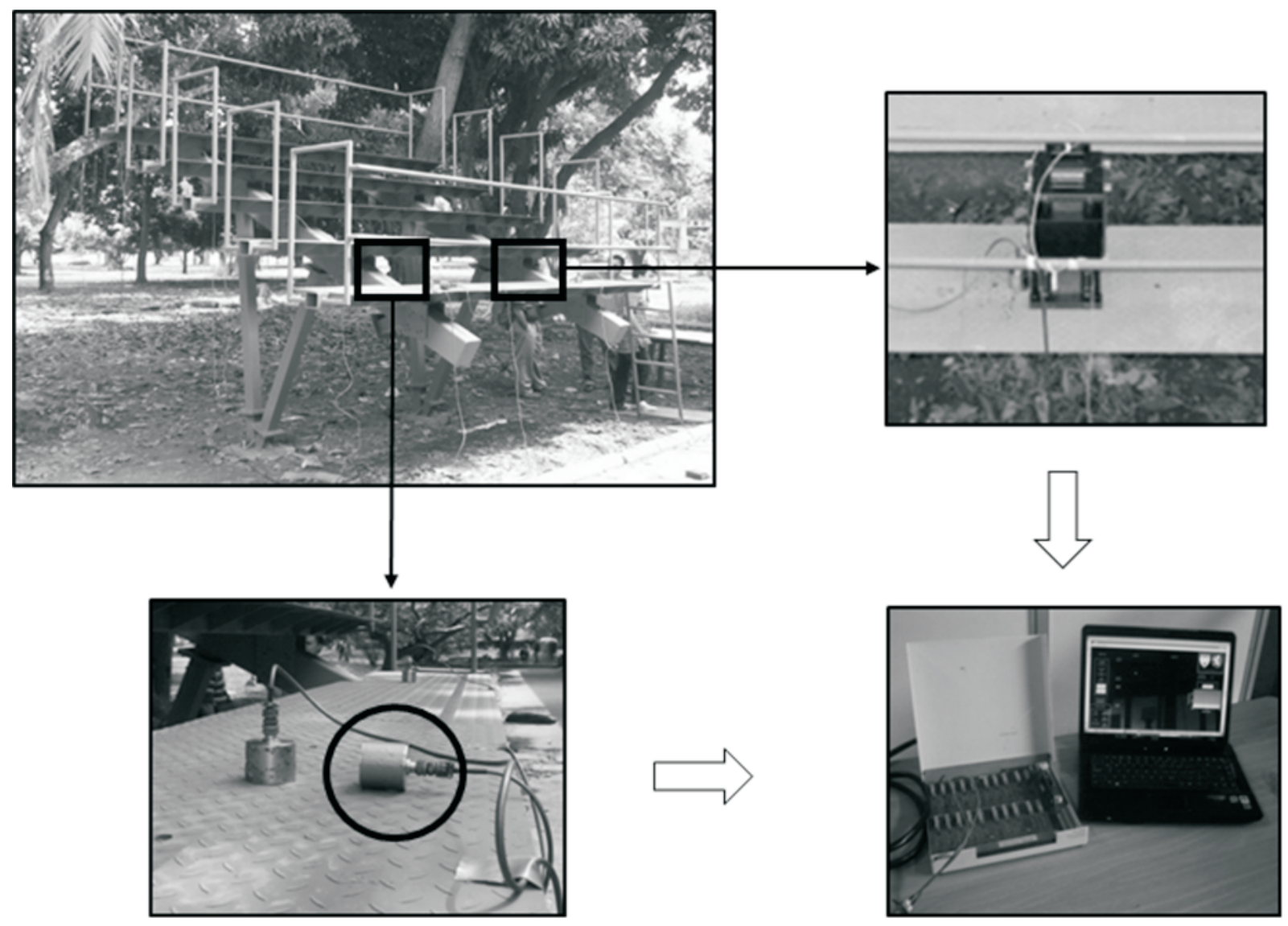

Figura 9. Instrumentación experimental

\subsubsection{Tribuna sin personas}

Las funciones de transferencia encontradas para la tribuna sin personas incluyendo la masa del actuador se muestran en la Figura 10. En los tres ensayos realizados se observa claramente la presencia de dos modos dinámicos en las frecuencias de $2.94 \mathrm{~Hz}$ y $4.45 \mathrm{~Hz}$, los cuales corresponden a un modo de flexión y un modo de torsión respectivamente. Adicionalmente, se realiza un ensayo de vibración libre con el objetivo de encontrar el factor de amortiguamiento modal de la estructura vacía, el cual corresponde a $0.33 \%$.

\subsubsection{Tribuna con una persona}

Se realizan tres ensayos con una persona sentada ubicada en tres diferentes posiciones, las cuales corresponden al primer peldaño, tercer peldaño y quinto peldaño. Igualmente, se realizan otros tres ensayos con una persona parada en las mismas ubicaciones anteriores. La Figura 11 muestra los resultados de los sistemas identificados en estas condiciones de ocupación, donde la línea sólida corresponde a los sistemas con la persona sentada y la línea punteada a los sistemas con la persona parada.

\subsubsection{Tribuna con dos y tres personas}

De igual forma, se realizan diferentes ensayos cuando la tribuna está ocupada por dos y tres personas ubicadas en diferentes posiciones. Para estas condiciones la Figura 12 muestra los sistemas dinámicos identificados, donde la línea sólida corresponde a la tribuna con dos personas y la línea punteada corresponde a la tribuna con tres personas. 
FUNCIÓN DE TRANSFERENCIA üe $/ \ddot{u}_{\mathrm{s}}$ : TRIBUNA SIN PERSONAS
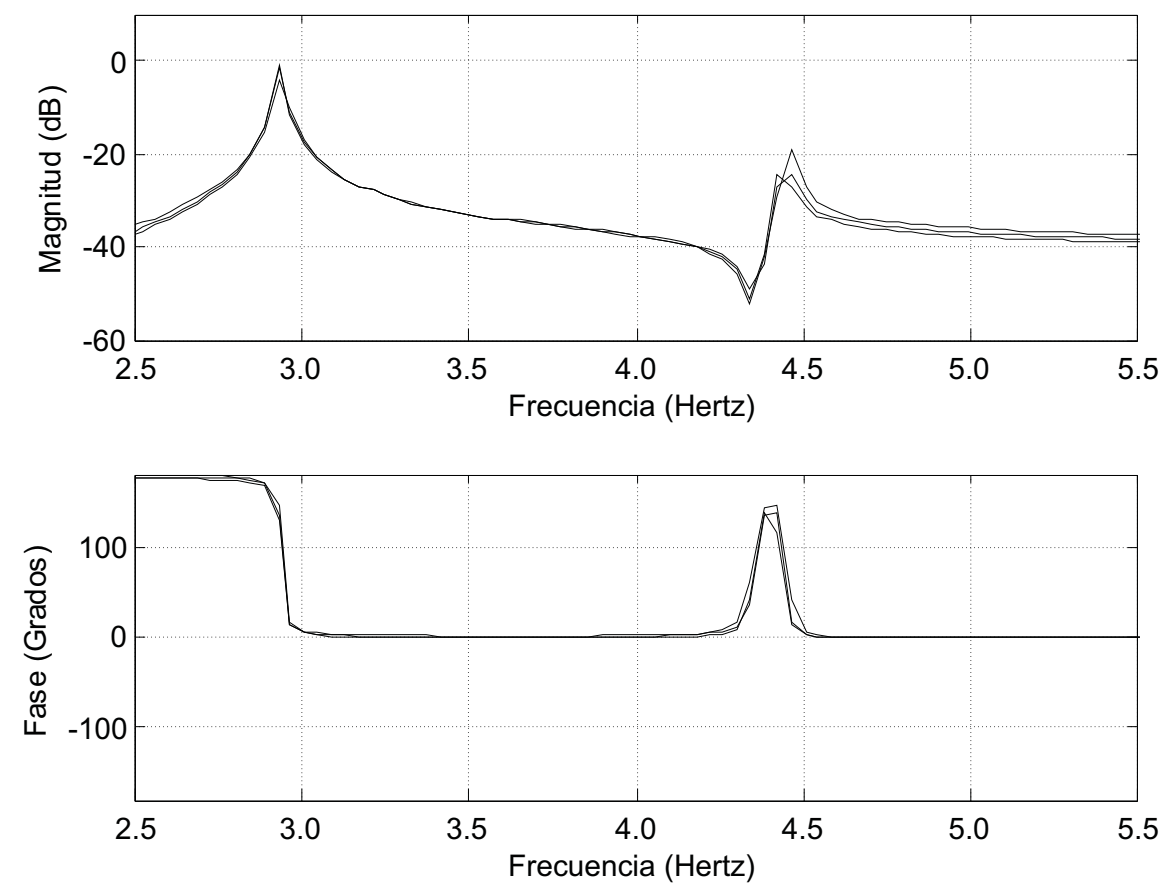

Figura 10. Funciones de transferencia de estructura sin personas

FUNCIÓN DE TRANSFERENCIA ü ${ }_{\mathrm{e}} / \ddot{u ̈}_{\mathrm{s}}:$ TRIBUNA CON 1 PERSONA
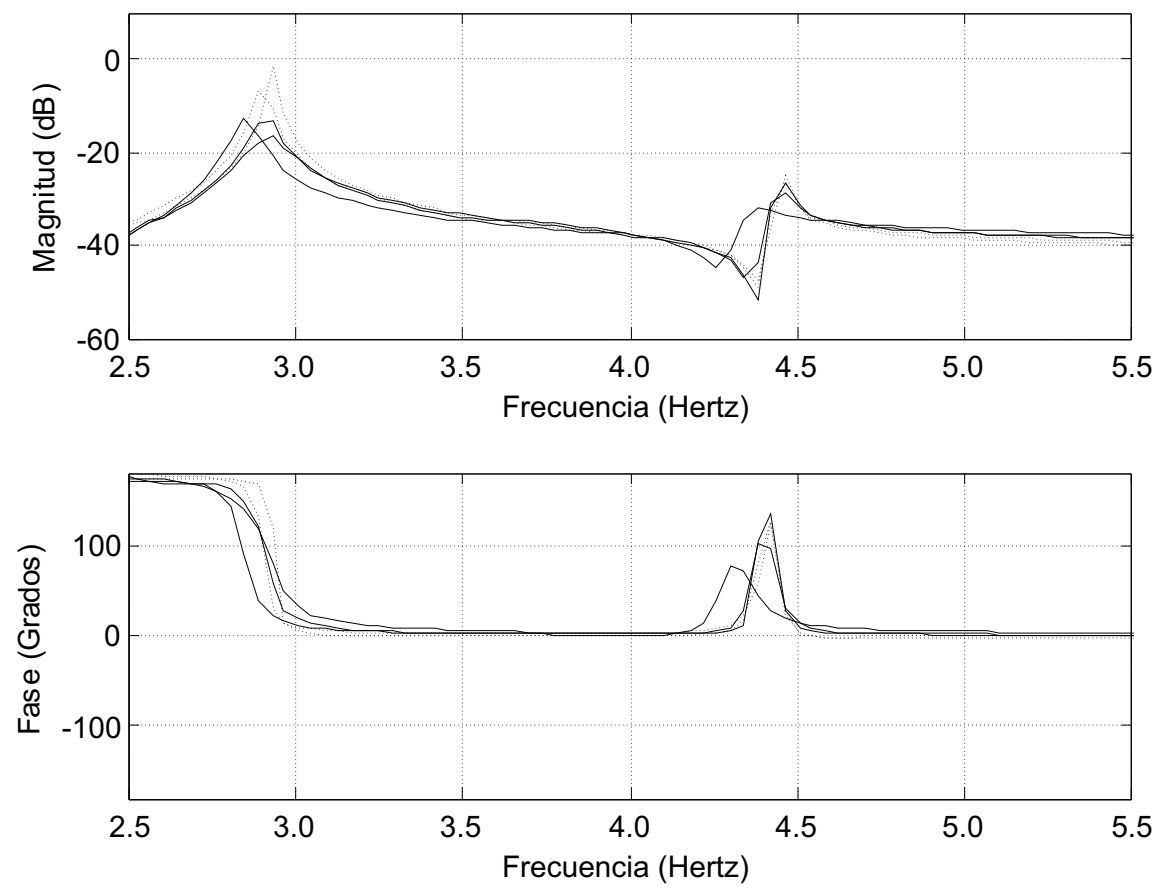

Figura 11. Funciones de transferencia de estructura con una persona sentada (línea sólida) y una persona parada (línea punteada) 
FUNCIÓN DE TRANSFERENCIA üe ë $_{s}$ : TRIBUNA CON 2 y 3 PERSONAS
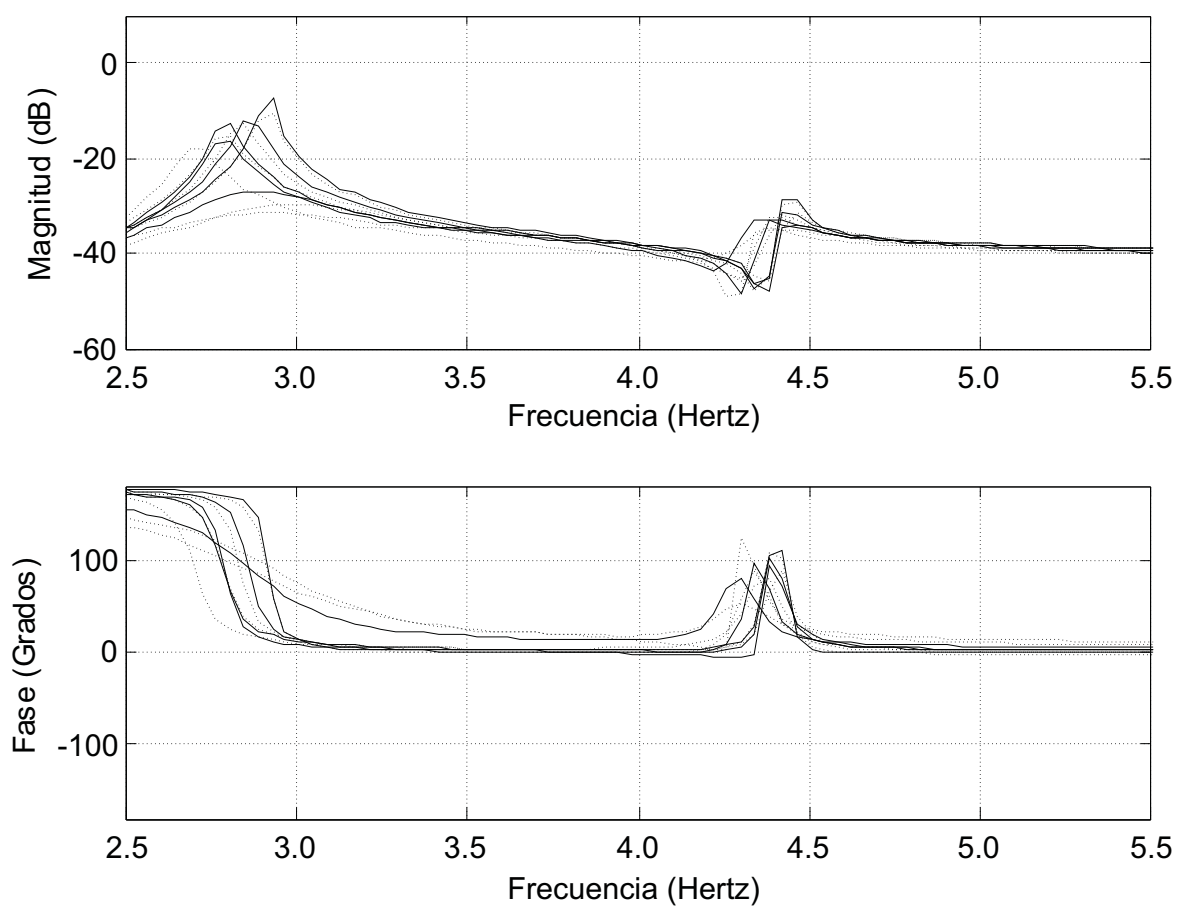

Figura 12. Funciones de transferencia de estructura con dos personas (línea sólida) y tres personas (línea punteada)

\subsection{Modelo dinámico}

Se construye un modelo analítico calibrado en un software comercial de análisis de estructuras para la tribuna sin personas. Sin embargo, debido a que la mayoría de los programas comerciales de elementos finitos son cajas negras que no permiten modificaciones ni la inclusión de nuevos códigos para implementar nuevas metodologías, como el caso particular de modelación de incertidumbre, se decide utilizar la herramienta ESTRULAB desarrollada en el programa matemático MATLAB por la Escuela de Ingeniería Civil y Geomática de la Universidad del Valle y el Departamento de Ingeniería Civil y Ambiental de la Universidad de South Carolina (Caicedo et al. 2005). Extrayendo las propiedades geométricas, información de materiales y tipos de elementos estructurales del software comercial, se pueden obtener las matrices de masa, rigidez y amortiguamiento necesarias para la formulación del modelo dinámico en el espacio de estados como se expresa en la Ec. (2).

\subsection{Modelo dinámico con incertidumbre paramétrica en el espacio de estados}

El modelo con incertidumbre se genera al asociar operadores de incertidumbre al modelo nominal descrito anteriormente. Al introducir incertidumbres paramétricas a las matrices de masa, rigidez y amortiguamiento se describen errores y variaciones no modeladas en elementos y coeficientes específicos en el sistema de ecuaciones dinámicas, los cuales pueden expresarse en LFTs como en la Figura 13.

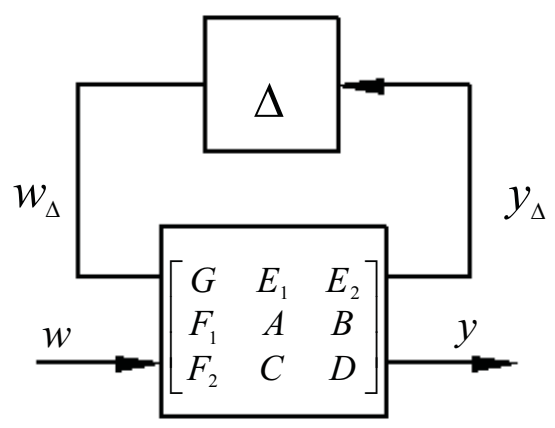

Figura 13. Configuración general para sistema con incertidumbre 
Donde $w_{\Delta}$ y $y_{\Delta}$ representan las entradas y salidas de incertidumbre, respectivamente, así como $y$ y $w$ representan las salidas y entradas del sistema, $\mathrm{y}$ las matrices $G, E_{1}, E_{2}, F_{l}, F_{2}$ relacionan las variables de incertidumbre relativa $r_{m}, r_{c} \mathrm{y} r_{k}$ con el sistema nominal descrito por las matrices $A, B$, $C, D$. Por último, la matriz $\Delta$ representa la incertidumbre del sistema, organizada de la siguiente forma:

$$
\begin{gathered}
\Delta=\left[\begin{array}{ccc}
\delta_{m} I_{m} & 0 & 0 \\
0 & \delta_{c} I_{c} & 0 \\
0 & 0 & \delta_{k} I_{k}
\end{array}\right] \\
-1 \leq \delta_{m}, \delta_{c}, \delta_{k} \leq 1
\end{gathered}
$$

Basándose en los modelos identificados y las condiciones de ocupación en que fueron realizados los ensayos, se definen los valores de incertidumbre relativa para el parámetro de masa, amortiguamiento y rigidez, los cuales corresponden a $r_{m}=0.2, r_{c}=0.95$ y $r_{k}=0$.Aquí se observa una alta variación en el factor de amortiguamiento, lo cual es producido cuando los ensayos se realizan con personas en posición sentada.

La Figura 14 muestra los resultados obtenidos del modelo analítico con incertidumbre cuando se tienen bloques de incertidumbre que afectan los parámetros de masa y amortiguamiento del sistema. Adicionalmente, la Figura 15 muestra las envolventes máxima y mínima obtenidas del modelo analítico, para comparar estos resultados con los modelos identificados experimentalmente, observándose que los sistemas identificados se encuentran incluidos dentro del conjunto de modelos descritos.

\section{Conclusiones}

Mediante registros de aceleración producidos por ensayos de vibración forzada para diferentes condiciones de ocupación de la estructura en estudio, se pudo identificar satisfactoriamente funciones de transferencia cuando su ocupación era hasta de tres personas. Estos resultados evidencian claramente las variaciones que las personas producen sobre las propiedades dinámicas, observándose que la mayor variación en frecuencias naturales es debida al incremento de la masa en la estructura, la cual está representada por el peso de las personas. De igual forma, se observa que la variación del amortiguamiento en la estructura está controlada por la posición de las personas, bien sea sentadas o paradas.
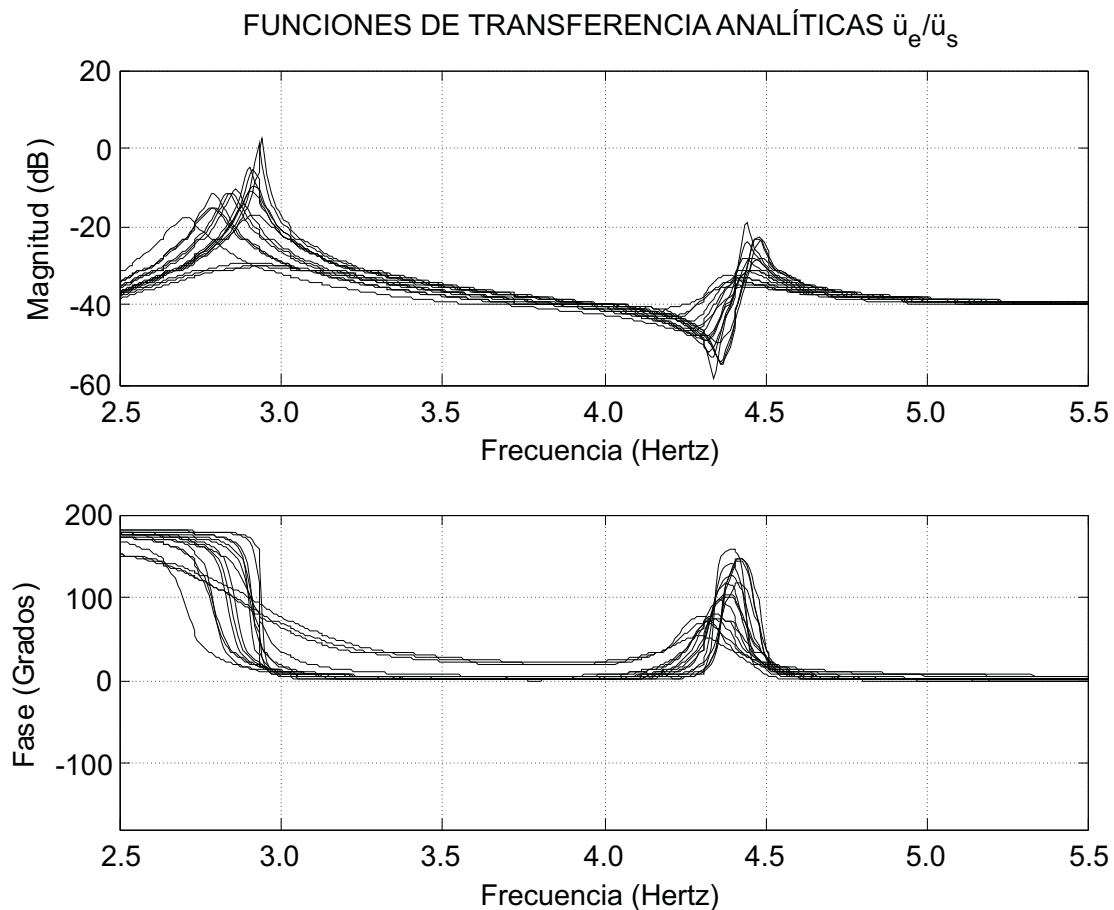

Figura 14. Funciones de transferencia del modelo analítico con incertidumbre en parámetros de masa y amortiguamiento 

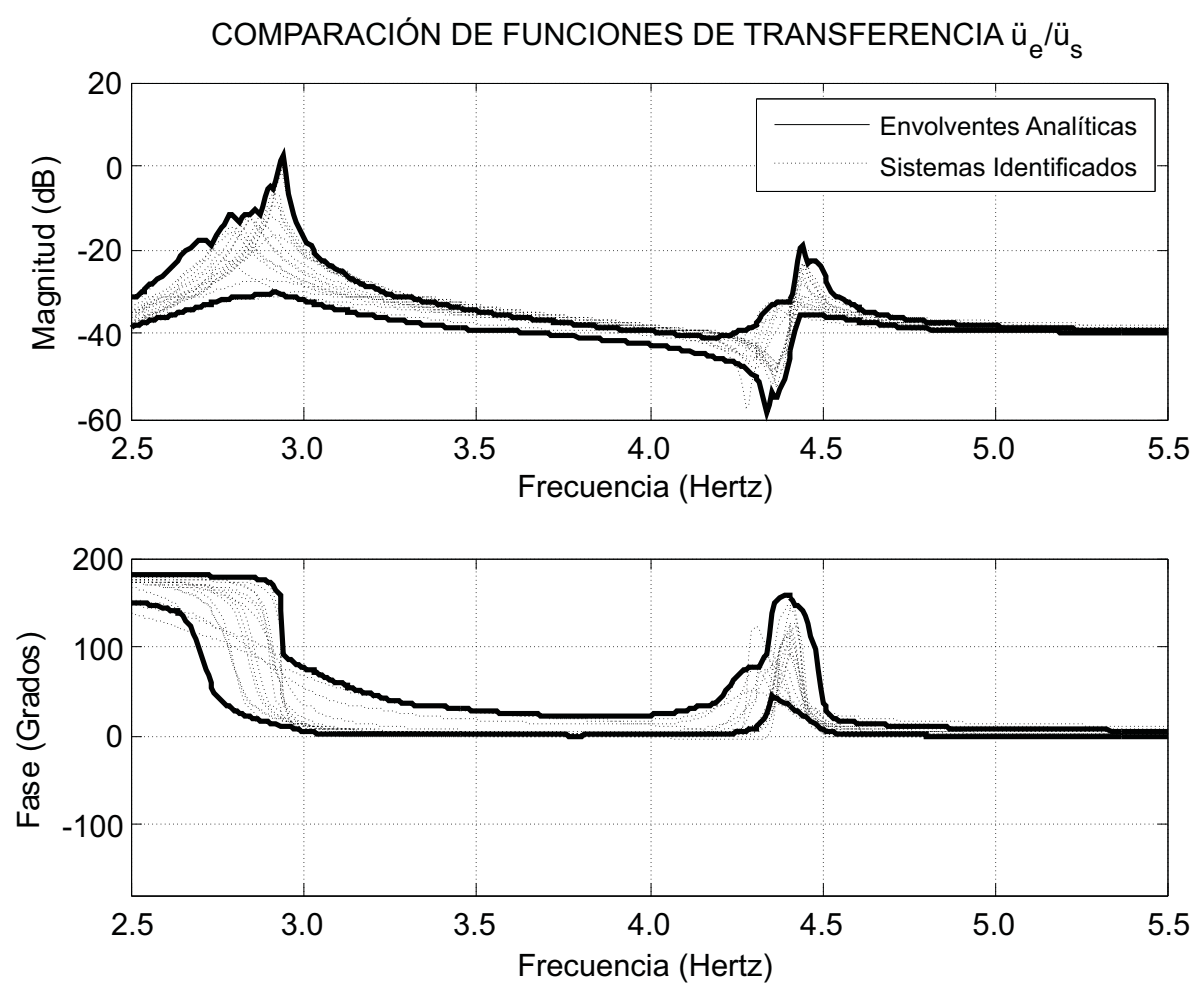

Figura 15. Comparación de resultados de modelo analítico y sistemas identificados experimentalmente

Surge entonces la necesidad de formular un modelo analítico con incertidumbre que pueda cubrir el conjunto de modelos identificados experimentalmente, lo que se constituye en el objetivo principal del trabajo. Para esto, se propuso la introducción de variaciones paramétricas en un modelo analítico calibrado y se describieron diferentes bloques de incertidumbre para cada uno de los casos de ocupación ensayados. De aquí se observa que es posible representar un conjunto de modelos identificados experimentalmente a partir de un modelo nominal analítico. Sin embargo cabe anotar que, aunque la incertidumbre paramétrica resulta fácil de comprender por estar relacionada directamente con los parámetros físicos del sistema, requiere un gran esfuerzo para su modelación al tratar con bloques de incertidumbre repetitivos de magnitud considerable, que para el caso particular presentado donde se toman variaciones en nueve grados de libertad, el bloque de incertidumbre generado es $\Delta \in \mathfrak{R}^{81 \times 81}$, lo cual hace su tratamiento matemático tedioso. Además de esto, se requiere un conocimiento detallado del sistema a modelar y no permite incorporar la dinámica no modelada de los sistemas.

\section{Agradecimientos}

Los autores agradecen la financiación de la Universidad del Valle y COLCIENCIAS mediante el programa Jóvenes Investigadores e Innovadores 2008 y el proyecto Investigación y desarrollo tecnológico de sistemas de monitoreo y control estructural para reducir las vibraciones producidas por la interacción humano/estructura (Contrato No. 003-2007).

\section{Referencias bibliográficas}

Balas, G. (1990). Robust Control of Flexible Structures: Theory and Experiments. Doctoral Thesis, California Institute of Technology, Pasadena, California. 
Balas, G., Doyle, J., Glover, K., Packard, A., \& Smith, R. (2001). m-Analysis and Synthesis Toolbox. For Use with MATLAB. MUSYN Inc. and The MathWorks, Inc.

Caicedo, J., Zarate, B., Marulanda, J., Thomson, P., \& Dyke, S. (2005). Desarrollo de una caja de herramientas para el análisis de estructuras por elementos finitos en MATLAB. En III Congreso Colombiano y VIII Seminario Internacional de Ingeniería Sísmica.

Chopra, A.K. (1995). Dynamics of Structures: Theory and Applications to Earthquake Engineering. New Jersey : Prentice Hall, Englewood Cliffs.

Gupta, S. (2000). Vibration Analysis of Structures Built up of Randomly Inhomogenieous Curved and Straight Beams using Stochastic Dynamic Stiffness Matrix Method. Bangalore: Indian Institute of Science.

Haldar, A., \& Sankaran, M. (2000). Realiability Assessment Using Stochastic Finite Element Analysis. New York: John Wiley \& Sons.

May, B.S. (1997) Probabilistic Robust Control: Theory and Applications. Doctoral Thesis, Earthquake Engineering Research Laboratory, California Institute of Technology, Pasadena.

Modarreszadeh, M. (2005) Dynamic Analysis of Structures with Interval Uncertainty. Doctoral Thesis, Department of Civil Engineering, Case Western Reserve University, Cleveland.

Moncayo, H., Marulanda, J., \& Thomson, P. (2006), Modal identification and monitoring of human-induced vibration of Cali's Olympic Stadium. En XXIII Southeastern Conference on Theoretical and Applied Mechanics (SECTAM XXIII).

Monte, L. (2009). Multi-model approach and evaluation of the uncertainty of model results. Rationale and applications to predict the behavior of contaminants in the abiotic components of the fresh water environment. Ecological Modelling, 220(12), 1469-1480.
Moreno, C.P., \& Thomson, P. (2010). Design of an optimal tuned mass damper for a system with parametric uncertainty. Annals of Operations Research.

Ortíz, A., Marulanda, J., \& Thomson, P. (2007). Caracterización del comportamiento dinámico de la tribuna occidental del estadio Pascual Guerrero durante un concierto musical. Ingeniería $y$ Competitividad, 9(2), 49-58.

Reynolds, P., Pavic, A., \& Ibrahim, Z. (2004). Changes of modal properties of a stadium structure occupied by a crowd. En 2nd international Modal Analysis Conference (IMAC XXII).

Skogestad, S., \& Postlethwaite, I. (2003). Multivariable Feedback Control: Analysis and design. New York: John Wiley \& Sons.

Trifunac, M. D., Ivanovic, S. S., \& Todorovska, M. I. (2001). Apparent Periods of a Building. I: Fourier Analysis, Journal of Structural Engineering, 127(5), 517-526.

Vélez, W., Gómez, D., \& Thomson, P. (2009). Ajuste de modelos de elementos finitos. Dyna: Revista de la Facultad de Minas. Universidad Nacional de Colombia, 76(158), 177-189.

Weinmann, A. (1991). Uncertain models and robust control. Vienna and New York: SpringerVerlag.

Zhuo, K., Doyle, J.C., \& Glover, K. (1991). Robust and Optimal Control. New Jersey: Prentice Hall. 